\title{
Links between central CB1-receptor availability and peripheral endocannabinoids in patients with first episode psychosis
}

\author{
Alex M. Dickens ${ }^{1,8 凶}$, Faith Borgan ${ }^{2,3,8}$, Heikki Laurikainen ${ }^{4,5,8}$, Santosh Lamichhane (iD) ${ }^{1}$, Tiago Marques (iD ${ }^{2,3}$, Tuukka Rönkkö ${ }^{1}$, \\ Mattia Veronese ${ }^{2,3}$, Tuomas Lindeman ${ }^{1}$, Tuulia Hyötyläinen ${ }^{6}$, Oliver Howes ${ }^{2,3}$, Jarmo Hietala (iD ${ }^{4,5}$ and Matej Orešič (iD) ${ }^{1,7 凶}$
}

There is an established, link between psychosis and metabolic abnormalities, such as altered glucose metabolism and dyslipidemia, which often precede the initiation of antipsychotic treatment. It is known that obesity-associated metabolic disorders are promoted by activation of specific cannabinoid targets (endocannabinoid system (ECS)). Our recent data suggest that there is a change in the circulating lipidome at the onset of first episode psychosis (FEP). With the aim of characterizing the involvement of the central and peripheral ECSs, and their mutual associations; here, we performed a combined neuroimaging and metabolomic study in patients with FEP and healthy controls (HC). Regional brain cannabinoid receptor type 1 (CB1R) availability was quantified in two, independent samples of patients with FEP $(n=20$ and $n=8)$ and HC $(n=20$ and $n=10)$, by applying three-dimensional positron emission tomography, using two radiotracers, $\left[{ }^{11} \mathrm{C}\right]$ MePPEP and $\left[{ }^{18} \mathrm{~F}\right] \mathrm{FMPEP}-\mathrm{d} 2$. Ten endogenous cannabinoids or related metabolites were quantified in serum, drawn from these individuals during the same imaging session. Circulating levels of arachidonic acid and oleoylethanolamide (OEA) were reduced in FEP individuals, but not in those who were predominantly medication free. In HC, there was an inverse association between levels of circulating arachidonoyl glycerol, anandamide, OEA, and palmitoyl ethanolamide, and CB1R availability in the posterior cingulate cortex. This phenomenon was, however, not observed in FEP patients. Our data thus provide evidence of cross talk, and dysregulation between peripheral endocannabinoids and central CB1R availability in FEP.

npj Schizophrenia (2020)6:21; https://doi.org/10.1038/s41537-020-00110-7

\section{INTRODUCTION}

Psychotic disorders are associated with a reduced life expectancy of $15-20$ years ${ }^{1}$, which is in part due to the high prevalence of cardiovascular disease, type 2 diabetes (T2DM) and metabolic syndrome ${ }^{2,3}$. Unhealthy lifestyles and pharmacological side effects have been suggested to be a major cause of these mortality rates ${ }^{4}$. However, abnormal glucose homeostasis, hyperinsulinemia, dyslipidemia, and accumulation of visceral fat are already evident in drug-naive first episode psychosis (FEP) patients, independent of obesity $^{5,6}$

There are many proposed mechanisms underlying the development of metabolic comorbidities. There is evidence that antipsychotic medication, particularly clozapine, leads to rapid weight gain ${ }^{7}$, while co-treatment with metformin can help reduce this $^{8}$. A recent study in FEP patients identified changes in specific circulating lipids, which are known to be associated with nonalcoholic fatty liver disease (NAFLD) and T2DM risk, prior to weight gain?

There is emerging evidence suggesting that the endocannabinoid system (ECS) might be dysregulated in various psychiatric disorders, including schizophrenia ${ }^{10-15}$. Specifically, in the cerebrospinal fluid (CSF), anandamide (AEA), and palmitoyl ethanolamide (PEA) were found elevated in schizophrenia as compared to controls $^{16}$, and AEA levels were inversely correlated to psychotic symptoms ${ }^{17}$. Furthermore, the ECS and related molecules have been shown to play a role in metabolic disorders ${ }^{18-20}$. Oleoylethanolamide (OEA) has been shown to directly alter lipid metabolism via peroxisome proliferator-activated receptor alpha $(\text { PPARa })^{21}$, resulting in anti-obesity properties, such as lower body weight, as well as decreased lipid levels in circulation and the liver $^{22}$. OEA was also found to be regulated by food intake in humans ${ }^{23}$. Taken together, these findings suggest that the dysregulation of the ECS could play a role in the development or progression of metabolic comorbidities observed in psychosis.

The ECS is composed of (1) cannabinoid receptors type 1 (CB1R) and type 2 (CB2R), (2) lipid-derived endocannabinoid ligands with affinity to these or other receptors, and (3) enzymes involved in the synthesis and degradation of these ligands ${ }^{24,25}$. The CB1Rs are found in the central nervous system (CNS), as well as in the periphery, throughout the gastrointestinal tract, liver, adipose tissue, and adrenal glands 26,27 . In the CNS, the CB1R plays an important role in cognition ${ }^{28}$, and has been implicated in mood and anxiety disorders ${ }^{29,30}$. Recent evidence from both our group and others suggests that central CB1R availability is altered psychosis ${ }^{31-33}$. We recently showed that CB1R availability is altered in patients with psychosis, where greater reductions in CB1R levels are associated with greater symptom severity and poorer cognitive functioning ${ }^{33}$. There is also evidence that endogenous cannabinoid ligands that act as CB1R agonists are elevated in the CSF of medication-naive psychotic patients ${ }^{11,14,16,17,34}$.

Metabolomics approaches have identified systemic metabolic changes in the context of psychosis ${ }^{9,35-37}$ and neurodegenerative disorders $^{38-40}$, suggesting that a strong link exists between the

\footnotetext{
${ }^{1}$ Turku Bioscience Centre, University of Turku and Åbo Akademi University, Turku, Finland. ${ }^{2}$ Department of Psychosis Studies, Institute of Psychiatry, Psychology \& Neuroscience, King's College London, London WC2R 2LS, UK. ${ }^{3}$ Psychiatric Imaging Group, MRC London Institute of Medical Sciences, Hammersmith Hospital, Imperial College London, London W12 OHS, UK. ${ }^{4}$ Department of Psychiatry, University of Turku, FI-20520 Turku, Finland. ${ }^{5}$ Turku PET Centre, Turku University Hospital, FI-20521 Turku, Finland. ${ }^{6}$ Department of Chemistry, Örebro University, 70281 Örebro, Sweden. ${ }^{7}$ School of Medical Sciences, Örebro University, 70281 Örebro, Sweden. ${ }^{8}$ These authors contributed equally: Alex M. Dickens, Faith Borgan, Heikki Laurikainen. ${ }^{凶}$ email: alex.dickens@utu.fi; matej.oresic@utu.fi
} 

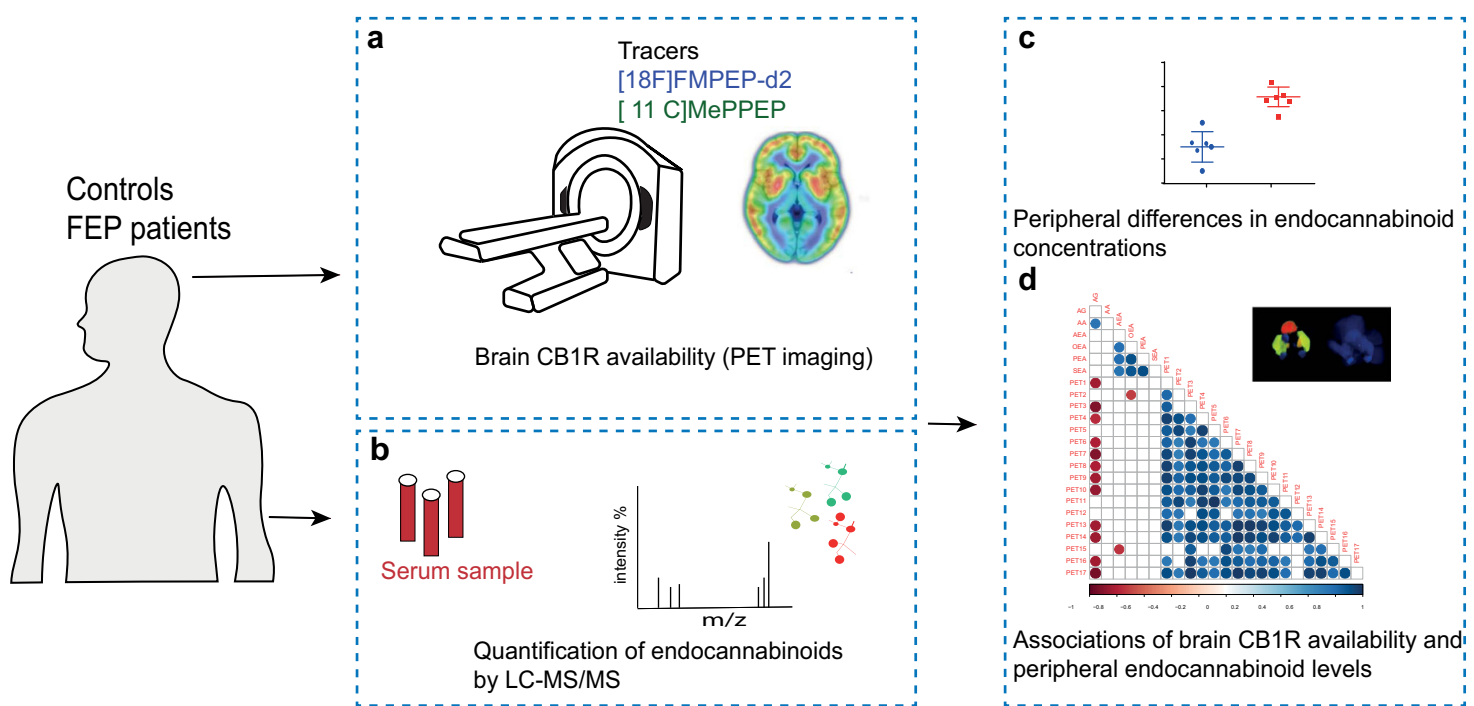

Fig. 1 An overview of the experimental design to study associations between brain CB1R availability and circulating endocannabinoids. a CB1R availability was investigated in vivo in male patients with, first episode psychosis (FEP) and healthy controls (HC) using a CB1R-selective radiotracer using positron emission tomography (PET), with arterial blood sampling. This study was performed at two study sites using two independent samples in the city of Turku, Finland, and an inner-city area of London, United Kingdom. A [ ${ }^{18}$ F]FMPEP-d2 PET tracer was used in Turku, while $\left[{ }^{11} \mathrm{C}\right]$ MePPEP tracer was used in London. $\mathbf{b}$ Quantification of circulating endocannabinoids was performed in matched FEP and HC subjects using a quantitative liquid chromatography-triple quadrupole mass spectrometry assay. c Peripheral differences in endocannabinoids between FEP and HCs were evaluated by univariate statistics. In order to assess if circulatory endocannabinoids associated with CB1R availability in the brain, we performed statistical correlation analysis between six endogenous endocannabinoids and 17 CB1R tracer distribution volumes. The correlation analysis was performed separately between FEP and HC for the two study sites.

circulating metabolome and diseases of the CNS. Several studies have demonstrated that psychotic patients have dysregulated metabolic profiles, such as dyslipidemia and dysglycemia ${ }^{41,42}$. Intriguingly, an imbalance of peripheral endocannabinoids has also been associated with various metabolic disorders, including diabetes and obesity ${ }^{43,44}$. Furthermore, increases in AEA, OEA, and PEA serum levels are associated with improvement in symptoms of psychosis ${ }^{45}$, which could imply that endocannabinoid signaling may not only be dysregulated in the CNS of psychotic patients, but also in the periphery ${ }^{12,14}$. However, to our knowledge, there are no data investigating the link between peripheral endocannabinoid levels and brain CB1R availability in healthy individuals or in FEP.

Therefore, we aimed to investigate the association between peripheral endocannabinoids and brain CB1R availability. To this end, we quantified serum endocannabinoids using liquid chromatography-triple quadrupole mass spectrometry (LC-QqQMS) and brain CB1R availability in a case-control setting, which included FEP patients and matched healthy controls $(\mathrm{HC}$; Fig. 1).

\section{RESULTS}

Levels of circulating endocannabinoids

We measured a total of ten endocannabinoids and related structures (Supplementary Table 1) from serum of both $\mathrm{HC}$ and FEP patients from the two study sites. Considerable increases in AG were observed in some samples, which can be explained by variable freezing times ${ }^{46}$. These samples were excluded from subsequent analyses, resulting in the following available sample numbers: Turku ( $n=10, \mathrm{HC} ; n=8, \mathrm{FEP})$ and London ( $n=11, \mathrm{HC}$; $n=15$, FEP). In the Turku study, there was a reduction in circulating levels of arachidonic acid (AA; 140.6 \pm 29.9 vs. $104.1 \pm 26.8 \mathrm{ng} / \mathrm{mL}$ ) and OEA (3.23 \pm 1.04 vs. $2.29 \pm 0.50 \mathrm{ng} / \mathrm{mL})$ in the FEP patients (Fig. 2a). AA forms the backbone of two major endocannabinoids $A E A^{47}$, and is released when they are broken down by fatty acid amide hydrolase ${ }^{48}$ and monoacylglycerol lipase ${ }^{49}$. In line with this, there is a trend toward an increase in AG observed in the FEP group $(2.32 \pm 0.94$ vs. $3.69 \pm 2.02 \mathrm{ng} / \mathrm{mL})$. Due to the use of serum, it was, however, not possible to differentiate between the levels of 1-arachidonoyl glycerol (1-AG) and 2-AG, because of their rapid isomerization at room temperature. Therefore, only the concentrations of total AG are reported.

The same endocannabinoids were, however, not altered in patients with FEP in the London study (Fig. 2b). In Turku, patients had already started antipsychotic medication, while in London, the patients were mostly drug naive or free of pharmacological treatments ${ }^{33}$. Previous literature has shown that endogenous central elevations in AEA (a CB1R and CB2R agonist, as well as a ligand to other proteins) found in patients with FEP ${ }^{14,17}$ are absent in patients taking typical antipsychotics, which agonize the actions of dopamine ${ }^{17}$. Thus, antipsychotic treatment in the Turku sample may account for the differences we observe. However, since the majority of the therapies involved atypical antopsychotics (Table 2 ), which were previously found not to normalize AEA levels ${ }^{17}$, this discrepancy could be due to other differences between the study populations, such as diagnosis, substance use, diet, or duration of illness. The full details of disease duration, medication, and diagnosis is provided in Tables 2 and 3.

Associations between central and peripheral ECSs

Given the known role of the ECS in dietary regulation ${ }^{50}$ and weight gain ${ }^{51}$, we next investigated the associations between peripheral endocannabinoid levels and CB1R availability in the ROls described in the methods. The results from the CB1-receptor availability study have previously been published ${ }^{33}$.

Initially, we explored how circulating levels of endocannabinoids are associated with CB1R availability in the brain in both studies using partial least squares regression (PLS-R), which allowed us to see how all of the endocannabinoids are associated with individual brain regions in one model. The $R^{2}$ values were increased in $\mathrm{HC}$ both in the Turku (Fig. 3a) and London (Fig. 3c) cohorts, as compared to the FEP group (Fig. 3b, Turku and Fig. 3d, London). We next explored the univariate associations between 

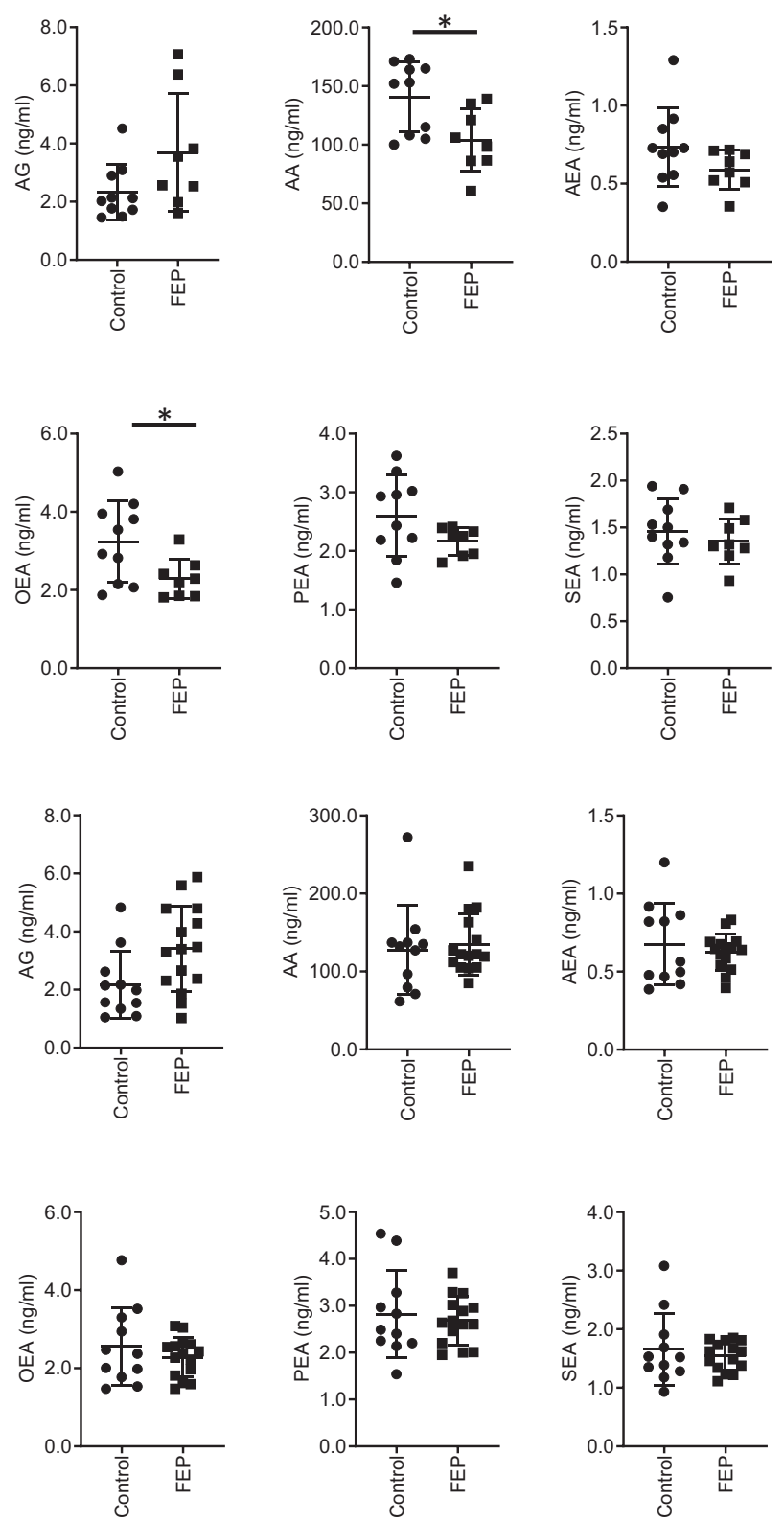

Fig. 2 Scatter plots of the levels of circulating endocannabinoids and related structures. The top six show the levels from the Turku cohort and the bottom 6 show the results from the KCL cohort. Key: ${ }^{*} p<0.05$.

levels of circulating endocannabinoids and CB1R availability in the brain. Positive correlations of CB1R availability between the brain regions were observed in $\mathrm{HCs}$ from both study sites (Fig. 3e). Similarly, levels of circulating endocannabinoids were also positively correlated with each other in the healthy subjects (Fig. 3e). Interestingly, the levels of peripheral endocannabinoids and central CB1R availability were, conversely, negatively correlated in HCs in both Turku and London samples (Fig. 3e). In FEP subjects, CB1R availability was also positively correlated between the different brain regions (Fig. 3f). However, the degree of positive correlation between the circulating endocannabinoids was reduced (Fig. $3 f$ ).

Notably, the negative correlations between CB1R availability and levels of circulating endocannabinoids, as observed in HC, were absent in FEP patients (Fig. 3e, f). The loss of this association is more pronounced in the Turku study (Fig. 4a, b) compared to the London study (Fig. 4c, d). This relative lack of association between central CB1R availability and circulating endocannabinoid levels in FEP suggests that there may normally be a functional link between these two systems, which may be dysregulated in FEP.

In order to further examine the associations between central CB1R availability and circulating peripheral endocannabinoids, we selected the brain region, from the available ROls, with the highest $R^{2}$ value from the PLS-R modeling, the posterior cingulate cortex (pcc; Fig. 3). The pcc has a critical role in a wide range of cognitive tasks $^{52}$ and is a major node in the default mode network ${ }^{53}$. Early changes have been observed in parts of the pcc during FEP ${ }^{54}$. For the majority of serum endocannabinoids measured in FEP patients, there was a clear loss of any negative correlation with CB1R availability, with few positive associations observed in the FEP patients (Fig. 5). This effect was more pronounced in the Turku study (Supplementary Fig. 1a), with similar trends observed in the London study, though these did not reach statistical significance (Supplementary Fig. 1b).

\section{DISCUSSION}

We demonstrated that the cross talk between the endocannabinoid networks in the brain and periphery are dysregulated in FEP, as compared to HC. Reduced levels of AA and OEA in FEP subjects were observed in the Turku study, but not in London, where the patients were predominately drug naive or free from pharmacological treatments. A negative association between brain CB1R availability in the majority of the ROls used in the positron emission tomography (PET) imaging and serum endocannabinoid levels was observed in healthy individuals, but not in FEP patients across the two independent cohorts of patients.

Endocannabinoids are lipid mediators with a vital role in the maintenance of metabolic and immune homeostasis ${ }^{55,56}$. Several studies have found that systemic lipid profiles are dysregulated in various psychotic disorders ${ }^{9,57}$. Our study could corroborate these earlier findings, suggesting that the systemic ECS is dysregulated in psychosis $12,34,58$. In the periphery, a significant drop in the circulatory endocannabinoid, AEA, was previously found to accompany the acute phase of schizophrenia ${ }^{12}$, although this finding could not be replicated in a larger cohort ${ }^{17}$. This is at odds with findings from the London study, as well as with the previous literature showing that patients with FEP not taking antipsychotics or using cannabis show increased levels of AEA in $\mathrm{CSF}^{14,17,34}$. However, the finding from the Turku study is consistent with previous work showing a downregulation of enzymes involved in the synthesis of endocannabinoid ligands in peripheral blood mononuclear cells of FEP patients, as compared to matched, $\mathrm{HC}^{58}$. However, small sample size in the Turku study may also be a contributing factor to the observed discrepancy. In addition to FEP, other neuroinflammatory diseases, including multiple sclerosis, Huntington's, and Parkinson's diseases, are characterized by ECS alterations in the periphery ${ }^{59}$.

Our previous data links rapid weight gain in FEP with elevated baseline levels of triglycerides of low carbon number and double bond count ${ }^{9}$, which are known to be associated with de novo lipogenesis, inhibition of lipolysis, and NAFLD ${ }^{60,61}$. Endocannabinoids are known to stimulate de novo lipogenesis and obesity ${ }^{62}$. In our previous hepatic venous catheterization study in humans, we found elevated liver fat to be associated both with increased levels of 2-AG, as well as higher splanchnic production of triglycerides of low carbon number and double bond count, suggesting that the ECS could contribute to the development of human NAFLD by affecting hepatic lipid metabolism ${ }^{60}$.

OEA is the monounsaturated analog of AEA, but, unlike AEA, acts independently of the cannabinoid pathway, with no reported direct activity at the two known cannabinoid receptors, CB1R and 
a
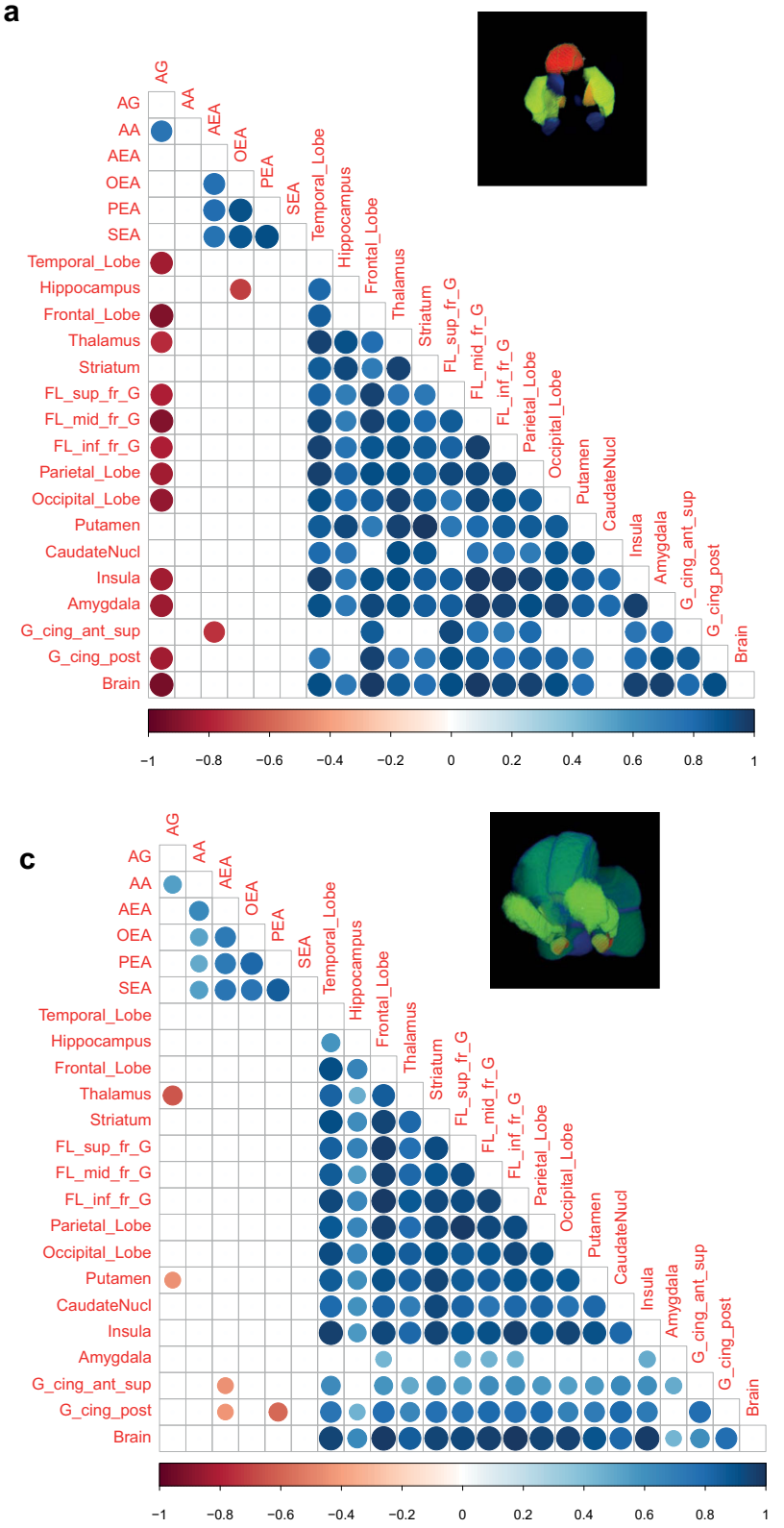

b
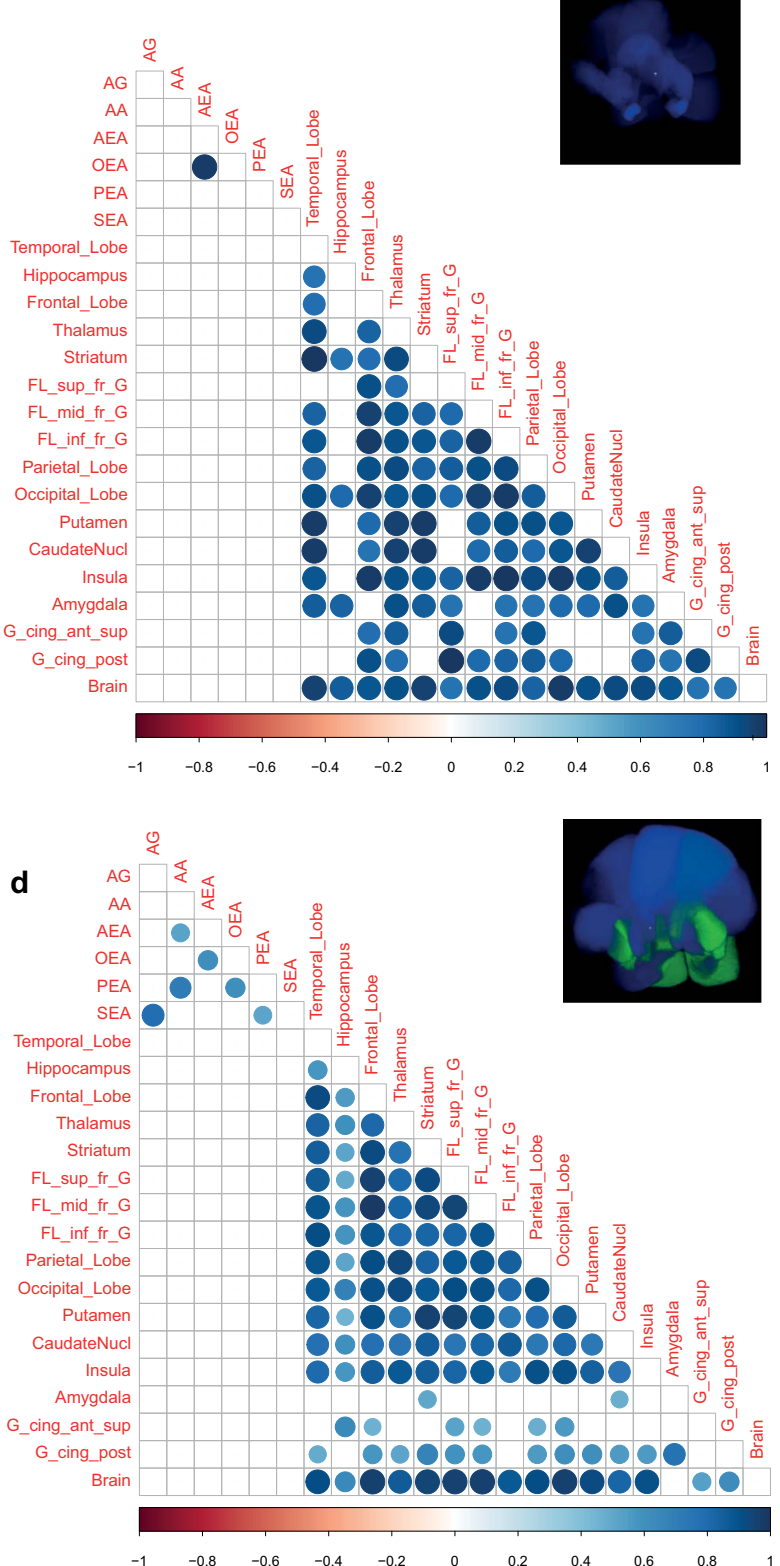

Fig. 3 Correlation analysis, performed between peripheral endocannabinoid concentrations and the distribution volumes of brain CB1R availability separately for patients with FEP and HC. Correlation brain maps where the $R^{2}$ values from the PLS regression analysis are projected into the 3D brain regions used for the PET analysis in the healthy controls (a, Turku, c, London) and FEP patients (b, Turku, $\mathbf{d}$, London). Red shading denotes high $R^{2}$, blue low $R^{2}$. e Spearman correlation coefficients between CB1R availability and circulating endocannabinoids in HC from both Turku and London. The CB1R availability was combined by first autoscaling the data from each site. f Spearman correlation coefficients between CB1R availability and circulating endocannabinoids in FEP from both Turku and KCL. The correlation coefficient values revealed positive correlations between CB1R availability in different regions of the brain in both FEP and HC. There was significant negative association between peripheral AEA and PEA concentrations, and CB1R availability in certain regions of the brain among $\mathrm{HCs}$, and this relationship was absent in FEP patients, at each respective site. Here, the blue gradient represents positive correlation, while the red gradient indicates negative correlation $(p$-values $<0.05)$.

$\mathrm{CB} 2 \mathrm{R}^{63}$. OEA stimulates fat utilization as a PPARa agonist ${ }^{64}$. Activation of PPARa by OEA is known to induce satiety and regulate body weight ${ }^{65}$. A study in mice also suggests that OEA inhibits food intake by recruitment of the brain histaminergic system $^{66}$. Our data suggest that decreased OEA in FEP subjects is likely the effect of antipsychotic meditation, as this was not seen in the cohort that was predominately unmedicated. However, there are other factors, such as disease duration and severity, which could also be a factor in the OEA difference between the sites. Inhibition of lipolysis due to diminished $\mathrm{OEA}^{65}$ may also contribute to increased liver fat, i.e., the accumulation of triacylglycerols in the liver, as observed by lipidomics in nonobese FEP patients who subsequently rapidly gained weight ${ }^{9}$. It is thus plausible that OEA levels are an early marker of propensity for FEP patients to gain weight, possibly as a result of therapy. This hypothesis will clearly need to be examined prospectively, in a larger study setting.

Our study also shows that peripheral endocannabinoids and central CB1R availability are inversely correlated in healthy individuals, while no such association is observed in FEP patients. 

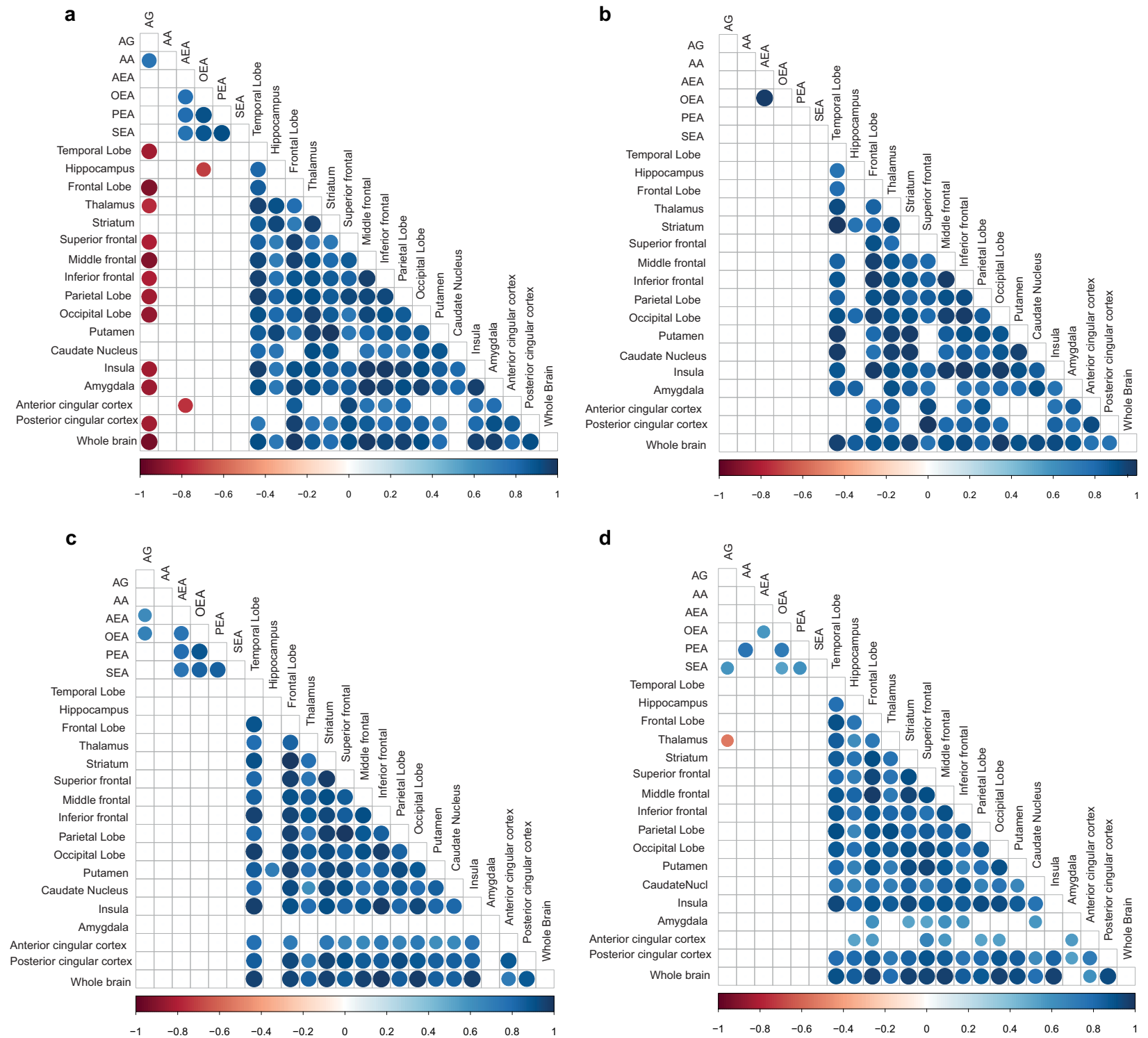

Fig. 4 Correlation analysis, performed between peripheral endocannabinoid concentrations and the distribution volumes of brain CB1R availability separately between FEP patients HC at the two study sites. a Spearman correlation coefficients between CB1R availability and circulating endocannabinoids in HC from the city of Turku. b Spearman correlation coefficients between CB1R availability and circulating endocannabinoids in FEP from the city of Turku. The correlation coefficient values revealed positive correlation between CB1R availability in different regions of the brain in both FEP and HC. c Spearman correlation coefficients between CB1R availability and circulating endocannabinoids in HC from an inner-city area of London. $\mathbf{d}$ Spearman correlation coefficients between CB1R availability and circulating endocannabinoids in FEP from inner-city area of London. There was significant negative association between peripheral arachidonoyl glycerol $(1+2)$ concentrations and CB1R availability in certain regions of the brain among HCs, which was absent in FEP patients, at different sites, respectively. Here, the blue gradient represents positive correlation, while the red gradient indicates negative correlation $(p$-values $<0.05)$.

The CB1R modulates energy homeostasis via both central and peripheral mechanisms ${ }^{67}$. The association between peripheral and central ECS measures suggests common systemic regulation of ECS in healthy individuals, which, however, is absent in FEP. Our findings from the Turku cohort indicate that both central and peripheral measures of the ECS were altered in patients with affective/non-affective FEP who were taking antipsychotic medication. In contrast, our findings from the London cohort indicate that patients who were predominately medication naive/free from pharmacological treatments, show central alterations in $\mathrm{CB} 1 \mathrm{R}$ availability without any alterations in peripheral levels of endocannabinoids. This suggests that peripheral measures of endocannabinoids may not be a useful biomarker for indexing central endocannabinoid dysfunction in FEP, alternatively, this negative finding may also be due to the small sample size, or differences in sample clinical characteristics between studies, including diagnosis, illness severity, therapies, and illness duration. This is in contrast to other findings in the field, where AEA has been suggested as a potential biomarker for schizophrenia ${ }^{16}$. The majority of the subjects in our study were confirmed to have a diagnosis of non-affective psychosis at 1-year follow up (Tables 2 and 3 ). There were, however, three cases of affective psychosis in the Turku cohort. This is too small of a group to explore if these individuals had an impact on the results.

Taken together, our data provides evidence of ECS dysregulation in FEP both centrally ${ }^{33}$ and in the periphery, in medicated patients with FEP. Our findings should be considered as preliminary due to our small sample size and partial inconsistency 

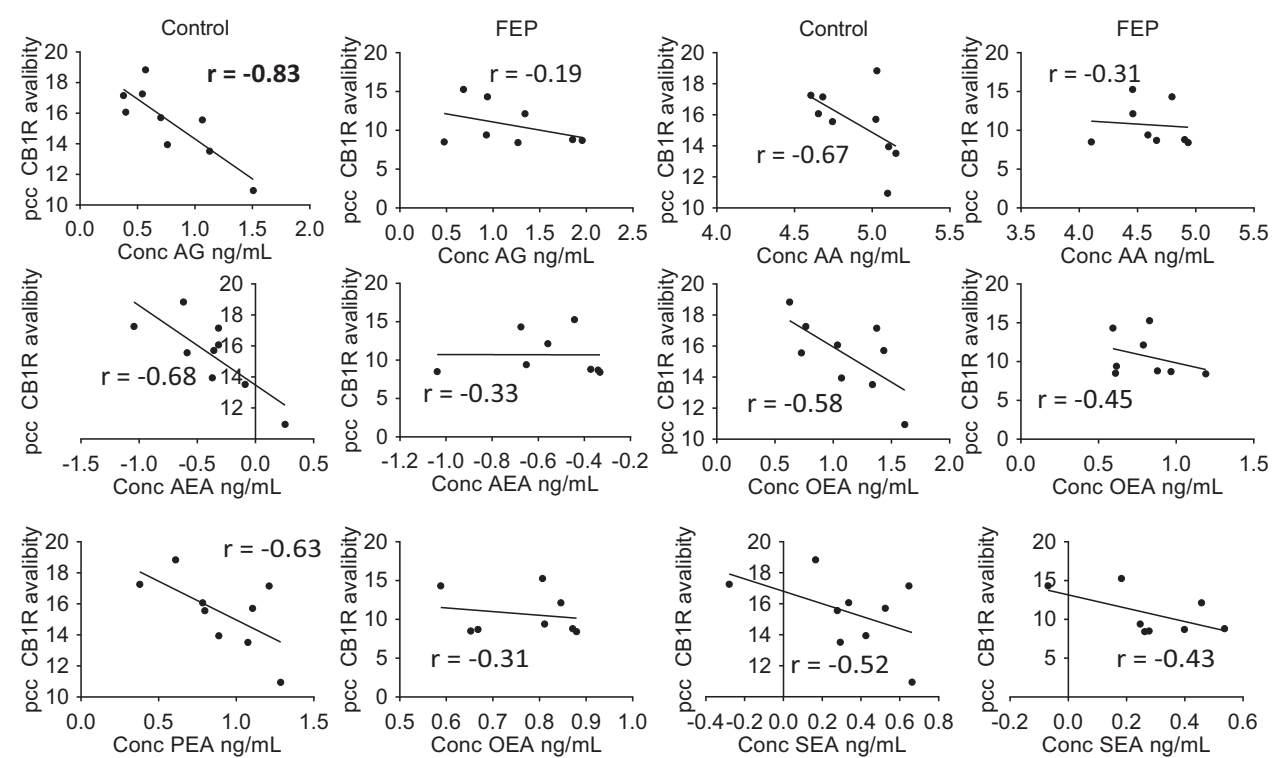

Fig. 5 Scatter plots fitted with a linear regression model of CB1R availability in the posterior cingulate cortex (PCC), versus the logtransformed circulating levels of endocannabinoids. The data was combined data from both studies, scaled to zero mean and unit variance within each study separately. The line shows the linear model for each dataset. Bold highlights of the $r$ value represent significant correlations $p<0.05$.

of results. Further studies are clearly needed in order to further examine the cross talk between the peripheral and central ECSs in health and psychosis.

\section{METHODS}

Ethics statement

Ethical approvals were obtained from respective study sites in Finland (ETMK 98/180/2013) and England (14/LO/1289). Subjects' capacities for consent were assessed and informed, written consent was obtained from all volunteers.

\section{Study design and participants}

The study setting was described in detail previously ${ }^{33}$. CB1R availability was investigated at two PET centers using independent samples; Turku $(n=11, \mathrm{HC} ; n=8, \mathrm{FEP})$ and London ( $n=23, \mathrm{HC} ; n=20$, FEP). However, some subjects were excluded due to increases in total arachidonoyl glycerol levels, caused by sample handling, resulting in the following subjects being included in the analysis: Turku $(n=10, \mathrm{HC} ; n=8$, FEP) and London ( $n=15, \mathrm{HC} ; n=11$, FEP; Table 1). Given putative sex differences in CB1R availability ${ }^{46}$, we only investigated males to remove sex as a source of variability. In Turku, the FEP patients were not antipsychotic naive (Table 2). Corresponding studies in females are currently ongoing.

FEP patients met the following inclusion criteria: (i) DSM-IV diagnosis of a psychotic disorder, determined by the SCID-I/P; (ii) illness duration of $<3$ years; and (iii) male sex. In Turku, FEP volunteers were already taking antipsychotic medication prior to the start of the study and had diagnoses of affective or non-affective psychosis. In London, FEP volunteers were, with the exception of two individuals, medication free from all pharmacological treatments for at least 6 months and had diagnoses of schizophrenia or schizoaffective disorder (DSM-IV criteria; Table 3). Healthy volunteers had no current/lifetime history of any Axis-I disorder as determined by the SCID-I/P, and were matched for age (age \pm 3 years) and sex (male). Exclusion criteria for study and healthy volunteers were: (i) current/lifetime history of substance abuse/dependence; (ii) substance use within the last month; and (iii) positive screen on toxicology tests for cannabis and other substances.

\section{CB1R availability PET imaging}

The full methods for PET CB1R imaging were described in detail previously ${ }^{33}$. However, a concise description for both studies, Turku and London, is provided here.
Table 1. Demographic characteristics of the study populations.

\begin{tabular}{|c|c|c|c|c|}
\hline & \multicolumn{2}{|l|}{ Turku } & \multicolumn{2}{|l|}{$\mathrm{KCL}$} \\
\hline & $\begin{array}{l}\text { FEP } \\
n=8 \\
\text { Mean (SD) }\end{array}$ & $\begin{array}{l}\mathrm{HC} \\
n=10 \\
\text { Mean (SD) }\end{array}$ & $\begin{array}{l}\text { FEP } \\
n=15 \\
\text { Mean (SD) }\end{array}$ & $\begin{array}{l}\mathrm{HC} \\
n=11 \\
\text { Mean (SD) }\end{array}$ \\
\hline Age (years) & $26.4(3.6)$ & $27.18(5.9)$ & $27.1(5.1)$ & $26.6(6.9)$ \\
\hline BMI $\left(\mathrm{kg} / \mathrm{m}^{2}\right)$ & $28.2(6.9)$ & $25.3(3.7)$ & $25.9(5.2)$ & $26.3(4.1)$ \\
\hline Years of education & $13.3(1.3)$ & $15.7(3.2)$ & $\mathrm{n} / \mathrm{a}$ & $\mathrm{n} / \mathrm{a}$ \\
\hline $\begin{array}{l}\text { Illness duration } \\
\text { (months) }\end{array}$ & $5.4(6.8)$ & $\mathrm{n} / \mathrm{a}$ & $27.1(10.1)$ & $\mathrm{n} / \mathrm{a}$ \\
\hline BPRS total score sum & $65.3(17.6)^{*}$ & $30.6(2.4)^{*}$ & $\mathrm{n} / \mathrm{a}$ & $n / a$ \\
\hline $\begin{array}{l}\text { BPRS positive } \\
\text { score sum }\end{array}$ & $20.0(7.3)^{*}$ & $9.2(0.6)^{*}$ & $\mathrm{n} / \mathrm{a}$ & $\mathrm{n} / \mathrm{a}$ \\
\hline PANSS positive & $\mathrm{n} / \mathrm{a}$ & $\mathrm{n} / \mathrm{a}$ & $24.4(5.4)$ & $\mathrm{n} / \mathrm{a}$ \\
\hline PANSS negative & $\mathrm{n} / \mathrm{a}$ & $\mathrm{n} / \mathrm{a}$ & $42.5(9.0)$ & $\mathrm{n} / \mathrm{a}$ \\
\hline PANSS general & $\mathrm{n} / \mathrm{a}$ & $\mathrm{n} / \mathrm{a}$ & $42.53(9.0)$ & $\mathrm{n} / \mathrm{a}$ \\
\hline PANSS total & $\mathrm{n} / \mathrm{a}$ & $\mathrm{n} / \mathrm{a}$ & 90.7 (16.6) & $\mathrm{n} / \mathrm{a}$ \\
\hline
\end{tabular}

Turku study. $\left[{ }^{18}\right.$ F]FMPEP-d2 was synthesized as described previously ${ }^{47}$, with slight modifications. The radiochemical purity was $>95 \%$ and the molar radioactivity was $>500 \mathrm{GBq} / \mu \mathrm{mol}$ at the end of synthesis, resulting in an injected tracer mass $<200 \mathrm{ng}$.

Positron emission scans were done in three-dimensional (3D) list mode, in increasing frame duration for a total scan range of 0-120 min, with a brain-dedicated high-resolution research tomograph (ECAT HRRT, Siemens Medical Solutions, Kemnath, Germany), after a bolus injection of $\left[{ }^{18} \mathrm{~F}\right]$ FMPEP-d2 (201 $\pm 11.1 \mathrm{MBq})$. Attenuation correction of PET was applied using a ${ }^{137} \mathrm{Cs}$ point source. Arterial plasma activity was measured continuously for the first $3.5 \mathrm{~min}$, and discretely thereafter at 4.5, 7.5, $11,15,20,25,30,35,40,45,50$, and $60 \mathrm{~min}$. Arterial input was corrected for tracer metabolite activity measured using thin layer chromatography and digital autoradiography. The resulting metabolite-free arterial input was corrected for temporal delay between blood and PET tissue measurements. Motion correction between PET frames was done by realigning the 
Table 2. Disease duration and medication in the Turku cohort.

\begin{tabular}{lllll}
\hline Illness duration (mo) & Antipsychotic medication (p.o.) & CPZ eqv DDD (Leucht) & BPRS positive sum** & Diagnosis \\
\hline 4 & Risperidone $1 \mathrm{mg}$ & $60 \mathrm{mg}$ & 13 & Affective psychosis \\
2 & Aripiprazole $15 \mathrm{mg}$ & $300 \mathrm{mg}$ & 21 & Affective psychosis \\
6 & Risperidone $2 \mathrm{mg}$ & $120 \mathrm{mg}$ & 13 & Schizophrenia* \\
1 & Olanzapine $5 \mathrm{mg}$ & $150 \mathrm{mg}$ & 31 & Affective psychosis \\
3 & Perphenazine $8 \mathrm{mg}$ & $80 \mathrm{mg}$ & Schizophreniform \\
22 & Aripiprazole $15 \mathrm{mg}$ & $300 \mathrm{mg}$ & 26 & Schizophrenia \\
2 & - & - & 27 & Schizophrenia \\
3 & Aripiprazole $15 \mathrm{mg}+$ olanzapine $10 \mathrm{mg}$ & $600 \mathrm{mg}$ & 17 & Schizophrenia \\
\hline${ }^{*}$ Cannabis dependance disorder. & & \\
${ }^{* * B P R S}$ Brief Psychiatric Rating Scale. & & \\
Data for each patient listed as separate row. & & \\
\hline
\end{tabular}

Table 3. Details of disease duration and medication for $\mathrm{KCL}$ cohort.

\begin{tabular}{llccc}
\hline Illness duration (mo) & Antipsychotic medication (p.o.) & CPZ eqv DDD (Leucht) & PANSS total & Diagnosis \\
\hline 36 & - & 0 & 79 & Schizophrenia \\
36 & 2 & 60 & 75 & Schizophrenia \\
24.5 & - & 0 & 103 & Schizophrenia \\
30 & 2 & 60 & 103 & Schizophrenia \\
30 & - & 0 & 116 & Schizophrenia \\
36 & - & 0 & 103 & Schizophrenia \\
36 & - & 0 & 66 & Schizoaffective disorder \\
12 & - & 0 & 63 & Schizophrenia \\
24 & 2 & 60 & 90 & Schizophrenia \\
12 & - & 0 & 69 & Schizoaffective disorder \\
36 & - & 0 & 107 & Schizophrenia \\
36 & - & 0 & 88 & Schizophrenia \\
6 & - & 0 & 101 & Schizophrenia \\
24 & - & 0 & 79 & Schizophrenia \\
28 & - & 0 & & Schizophrenia \\
\hline Data for each patient listed as separate row. & & & \\
\hline
\end{tabular}

PET frames to a single reference frame with the most uptake on average. Whole-brain T1-weighted MRI images were acquired by a Philips 3T Ingenuity PET/MR hybrid scanner. The individual T1-weighted images were co-registered to the sum of the realigned PET frames. Inverse normalization parameters obtained from normalizing the co-registered T1weighted images to MNI space were used to fit the Hammersmith anatomical atlas to the PET frames (Hammers et. al 2003). Data preprocessing were performed using Statistical Parametric Mapping 12 (http://www.fil.ion.ucl.ac.uk/spm) and MATLAB R2014b (Mathworks Inc., Natick, MA).

London study. Continuous, 90-min PET scans were acquired on a PET/CT (Hi-Rez Biograph 6 CT44931; Siemens Medical Solutions, Kemnath, Germany) in 3D, after bolus injection of $314 \pm 34.4 \mathrm{MBq}$ of $\left[{ }^{11} \mathrm{C}\right] \mathrm{MePPEP}$, as previously described ${ }^{48-50}$. CT scans were acquired prior to each PET scan for correction of attenuation and scatter. Continuous arterial blood sampling took place for the first $15 \mathrm{~min}$ of the scan that was followed by discrete blood sampling at 2, 5, 10, 15, 20, 25, 35, 40, 50, 60, 70, 80, and $90 \mathrm{~min}$ after radioligand injection. Images were reconstructed with filtered back-projection, including corrections for attenuation and scatter. There were no significant group differences in injected mass, injected activity, or specific activity. To aid anatomical localization, each volunteer received a high-resolution structural 3D T1-weighted magnetic resonance scan acquired on a General Electric MR750 3 T scanner (MR750; GE Healthcare, Chicago, IL).

Data preprocessing was performed using a combination of Statistical Parametric Mapping 12 (http://www.fil.ion.ucl.ac.uk/spm) and FSL (http://www.fsl.fmrib.ox.ac.uk/fsl) functions, as implemented in MIAKAT (http://miakat.org). Motion correction was applied to non-attenuationcorrected images ${ }^{51}$. Non-attenuated-corrected frames were realigned to a single "reference" frame (corresponding to that with the highest number of counts) by employing a mutual information algorithm. The transformation parameters were then applied to the corresponding attenuated-corrected dynamic images, creating a movement-corrected dynamic image that was used for subsequent analysis. Realigned frames were then summated to create single-subject motion-corrected maps that were then used for MRI and PET co-registration, prior to PET data quantification. T1-weighted structural images were co-registered to the PET image using rigid body transformation. Normalization parameters were obtained by warping the co-registered structural MRI to MNI space (International Consortium for Brain Mapping ICBM/MNI) using biascorrected segmentation. The inverse of these parameters was used to fit a neuroanatomical atlas to each individual PET scan using the Hammersmith atlas ${ }^{52}$. Whole-blood time-activity curves (TACs) were fitted using a multi-exponential function as derived by Feng's model ${ }^{53}$. For each scan, a time delay was fitted and applied to the input functions 
(both parent and whole-blood TACs) to account for any temporal delay between blood sample measurement and target tissue data. Regions of interest (ROI) were harmonized with the Turku PET dataset.

In both Turku and London studies, CB1R availability was indexed using the volume of distribution (VT $\mathrm{ml} / \mathrm{cm} 3$ ) of the respective PET tracer, as calculated using the Logan graphical method with a metabolite-corrected arterial plasma input function. The resulting model was validated as described previously ${ }^{33,54}$. The temporal lobe, frontal lobe, anterior cingulate cortex, pcc, striatum, parietal lobe, occipital lobe, putamen, nucleus caudatus, insula, amygdala, and whole brain were chosen as ROI for PET. Hippocampal, amygdala, superior temporal gyral, superior frontal, middle frontal, inferior frontal, and orbitofrontal subregions were included to explore regional differences, within relevant ROI. Due to the use of different PET tracers used in both sites, the CB1R availability data were scaled to unit variance and zero mean within each study, when analyzed together.

\section{Analysis of serum endocannabinoids}

The following peripheral endocannabinoids and related structures were measured using a targeted LC-QqQMS method: THC-COOH, arachidonoyl ethanolamide (AEA), N-arachidonoyl dopamine (NADA), 2-AG, 1-AG, OEA, PEA, 2-arachidonoyl glycerol ether, $A A$, and stearoyl ethanolamide from the arterial serum collected during the PET imaging. The serum collection was unified at both sites, in order to allow for direct comparison of results. Serum samples were stored at $-80^{\circ} \mathrm{C}$ for 12.1 months ( \pm 8.72 months) in Turku study site and 24.9 months ( \pm 8.66 months) at KCL. The analytical method was developed for serum samples and all solvents were LC-grade (Sigma-Aldrich Inc., St. Louis, MO). Initially, the internal standards $(10 \mu \mathrm{L}$, $100 \mathrm{ng} / \mathrm{mL}$, of THC-COOH-d9, 2-AG-d5, NADA-d8, and AEA-d8 in EtOH) were added to $200 \mu \mathrm{L}$ of serum in silylated $2 \mathrm{~mL}$ glass chromatography vials. The proteins were then precipitated using acetonitrile $(400 \mu \mathrm{L})$ containing $0.1 \% \mathrm{v} / \mathrm{v}$ formic acid (LC-grade, Sigma-Aldrich). This was done in metal and glass syringes to ensure no contamination from plastic. The samples were then briefly vortexed and placed at $-20^{\circ} \mathrm{C}$ for $30 \mathrm{~min}$. The samples were then centrifuged $\left(10,000 \times g, 4^{\circ} \mathrm{C}\right)$ for $10 \mathrm{~min}$. The supernatant was then removed and placed in fresh silylated vials and stored for no more than $24 \mathrm{~h}$ before solid-phase extraction (SPE) of the endocannabinoids.

The SPE (reverse-phase HLB, $30 \mathrm{mg}$ sorbent) was performed in 96-well plates (Waters Inc., Milford, MA) and performed on ice to reduce plastic contamination. Initially, $1 \mathrm{~mL}$ of ultrapure $\mathrm{H}_{2} \mathrm{O}$ was added to the top of each well. The supernatant was then transferred into $\mathrm{H}_{2} \mathrm{O}$ via a glass Pasteur pipette, and gently mixed by pipetting up and down. The vacuum pump ( $-3 \mathrm{mbar}$ ) was then started and the wells allowed to dry. The wells were then washed twice with $15 \% \mathrm{ACN}$ in ultrapure $\mathrm{H}_{2} \mathrm{O}(500 \mu \mathrm{L}, 0.1 \% \mathrm{v} / \mathrm{v}$ formic acid). The pressure was then reduced to $-15 \mathrm{mbar}$ and the wells dried for $15 \mathrm{~min}$. The waste container under the 96 -well sorbet plate was then replaced with $750 \mu \mathrm{L}$ silylated glass insert vials in a deep 96-well plate. The endocannabinoids were eluted by allowing acetone $(350 \mu \mathrm{L}, 0.1 \% \mathrm{v} / \mathrm{v}$ formic acid) to pass through the sorbent bed twice. The samples were then evaporated to dryness under a stream of $\mathrm{N}_{2}$ prior to resuspension in a $70 \%$ solution of $\mathrm{ACN}(100 \mu \mathrm{L}, 30 \%$ ultrapure water, $1 \% \mathrm{v} / \mathrm{v}$ acetic acid). The samples were then vortexed briefly and stored at $-20^{\circ} \mathrm{C}$ prior to the LC-QqQMS experiment.

The endocannabinoids were separated using ultra-high-performance liquid chromatography. The two eluent solvents were (i) $\mathrm{H}_{2} \mathrm{O}(1 \% \mathrm{v} / \mathrm{v}$ acetic acid) and (ii) ACN. The gradient was set up as follows: the initial conditions were $70 \%$ B for $4.5 \mathrm{~min}$ increasing to $80 \%$ B by $7 \mathrm{~min}$. By $8 \mathrm{~min}$, the gradient was increased to $95 \% \mathrm{~B}$ and held there for $10 \mathrm{~min}$. The gradient was reduced to the starting conditions by $10.5 \mathrm{~min}$ and the column flushed with a minimum of ten column volumes. The flow rate was set at $0.5 \mathrm{~mL} /$ min throughout the run and the column temperature set to $60^{\circ} \mathrm{C}$. The column used for the separation was a Waters ACQUITY BEH reverse-phase C18 column $(130 \AA, 1.7 \mu \mathrm{m}, 2.1 \mathrm{~mm} \times 50 \mathrm{~mm})$. The injection volume was $10 \mu \mathrm{L}$. The needle was washed three times both before and after the injection with two solvents: (i) IPA:ACN (1:1) and (ii) $\mathrm{H}_{2} \mathrm{O}: \mathrm{MeOH}$ (1:1). The mass spectrometer 5500 QTRAP (AB Sciex Inc., Framingham, MA) was set up in scheduled multiple reaction monitoring (MRM) mode that allows for the setup of detection windows around the peak of interest to improve sensitivity. The details of the MRM transitions can be found in Supplementary Table 1 . The analytes were quantified using a six-point standard curve and, where possible, with the matched labeled standard. Where no labeled standard was available, we used the closest related molecule as the internal standard.

\section{Statistical analysis}

The nonparametric Mann-Whitney $U$ test was used for comparing the levels of circulating endocannabinoids using the log-transformed data, and performed using GraphPad Prism v. 7.04 (GraphPad Software Inc., San Diego, (A). In order to compare the association between circulating endocannabinoids with brain CB1R availability, two methods were utilized: (i) PLS-R modeling was used in order to combine multiple brain regions and circulating endocannabinoid measures. This analysis was performed using PLS-Toolbox v. 8.6 (Eigenvector Research Inc., Manson, WA) and MATLAB 2017b (Mathworks Inc., Natick, MA). Due to the small number of samples, a leave-one-out cross-validation, using the number of iterations of the smallest group size, was utilized to ensure model validity. (ii) Spearman correlation coefficients were calculated using the statistical toolbox in MATLAB 2017b and $p$-values $<0.05$ (two-tailed) were considered significant for the correlations. The individual spearman correlation coefficients $(R)$ were illustrated as a heatmap using the "corrplot" package for the $\mathrm{R}$ statistical programming language ${ }^{55}$. The individual correlations between the serum endocannabinoids and CB1R availability were plotted, and analyzed using GraphPad Prism.

\section{Generation of the statistical association brain maps}

In order to visualize the associations of CB1R availability across different brain regions, the $R^{2}$ values from the individual brain regions arising from the PLS-R modeling were mapped onto the same brain template as the PET image analysis, using MATLAB 2017b. The resultant image was then visualized using CARIMAS v. 2.9 (Turku PET Centre, Turku, Finland). The color scale for each brain region was defined by the $\mathrm{HC}$ in each cohort and then applied to the FEP patients.

\section{Reporting summary}

Further information on research design is available in the Nature Research Reporting Summary linked to this article.

\section{DATA AVAILABILITY}

The data that support the findings of this study are available from the corresponding author upon reasonable request.

\section{CODE AVAILABILITY}

The MATLAB scripts used for statistical analysis in this study are available from the corresponding author upon reasonable request.

Received: 28 January 2020; Accepted: 7 July 2020; Published online: 26 August 2020

\section{REFERENCES}

1. Saha, S., Chant, D. \& McGrath, J. A systematic review of mortality in schizophrenia: is the differential mortality gap worsening over time? Arch. Gen. Psychiatry 64, 1123-1131 (2007).

2. Ringen, P. A., Engh, J. A., Birkenaes, A. B., Dieset, I. \& Andreassen, O. A. Increased mortality in schizophrenia due to cardiovascular disease-a non-systematic review of epidemiology, possible causes, and interventions. Front. Psychiatry 5, 137 (2014).

3. Mukherjee, S., Schnur, D. B. \& Reddy, R. Family history of type 2 diabetes in schizophrenic patients. Lancet 1, 495 (1989).

4. Arango, C., Bobes, J., Kirkpatrick, B., Garcia-Garcia, M. \& Rejas, J. Psychopathology, coronary heart disease and metabolic syndrome in schizophrenia spectrum patients with deficit versus non-deficit schizophrenia: findings from the CLAMORS study. Eur. Neuropsychopharmacol. 21, 867-875 (2011).

5. Pillinger, T. et al. Impaired glucose homeostasis in first-episode schizophrenia: a systematic review and meta-analysis. JAMA Psychiatry 74, 261-269 (2017).

6. Pillinger, T., Beck, K., Stubbs, B. \& Howes, O. D. Cholesterol and triglyceride levels in first-episode psychosis: systematic review and meta-analysis. Br. J. Psychiatry 211, 339-349 (2017).

7. Covell, N. H., Weissman, E. M. \& Essock, S. M. Weight gain with clozapine compared to first generation antipsychotic medications. Schizophr. Bull. 30, 229-240 (2004).

8. Liu, Z. et al. Metformin for treatment of clozapine-induced weight gain in adult patients with schizophrenia: a meta-analysis. Shanghai Arch. Psychiatry 27, 331-340 (2015). 
9. Suvitaival, T. et al. Serum metabolite profile associates with the development of metabolic co-morbidities in first-episode psychosis. Transl. Psychiatry 6, e951 (2016).

10. Newell, K. A., Deng, C. \& Huang, X. F. Increased cannabinoid receptor density in the posterior cingulate cortex in schizophrenia. Exp. Brain Res. 172, 556-560 (2006)

11. Koethe, D. et al. Anandamide elevation in cerebrospinal fluid in initial prodromal states of psychosis. Br. J. Psychiatry 194, 371-372 (2009).

12. De Marchi, N. et al. Endocannabinoid signalling in the blood of patients with schizophrenia. Lipids Health Dis. 2, 5 (2003).

13. Parolaro, D., Realini, N., Vigano, D., Guidali, C. \& Rubino, T. The endocannabinoid system and psychiatric disorders. Exp. Neurol. 224, 3-14 (2010).

14. Leweke, F. M. et al. Anandamide levels in cerebrospinal fluid of first-episode schizophrenic patients: impact of cannabis use. Schizophr. Res. 94, 29-36 (2007)

15. Bioque, $M$. et al. Peripheral endocannabinoid system dysregulation in firstepisode psychosis. Neuropsychopharmacology 38, 2568-2577 (2013).

16. Leweke, F. M., Giuffrida, A., Wurster, U., Emrich, H. M. \& Piomelli, D. Elevated endogenous cannabinoids in schizophrenia. Neuroreport 10, 1665-1669 (1999).

17. Giuffrida, A. et al. Cerebrospinal anandamide levels are elevated in acute schizophrenia and are inversely correlated with psychotic symptoms. Neuropsychopharmacology 29, 2108-2114 (2004).

18. lannotti, F. A., Di Marzo, V. \& Petrosino, S. Endocannabinoids and endocannabinoid-related mediators: targets, metabolism and role in neurological disorders. Prog. Lipid Res. 62, 107-128 (2016).

19. Matias, I. \& Di Marzo, V. Endocannabinoids and the control of energy balance. Trends Endocrinol. Metab. 18, 27-37 (2007).

20. Di Marzo, V., Piscitelli, F. \& Mechoulam, R. Cannabinoids and endocannabinoids in metabolic disorders with focus on diabetes. Handb. Exp. Pharmacol. 203, 75-104 (2011).

21. Lo Verme, J. et al. Regulation of food intake by oleoylethanolamide. Cell Mol. Life Sci. 62, 708-716 (2005).

22. Fu, J., Oveisi, F., Gaetani, S., Lin, E. \& Piomelli, D. Oleoylethanolamide, an endogenous PPAR-alpha agonist, lowers body weight and hyperlipidemia in obese rats. Neuropharmacology 48, 1147-1153 (2005)

23. Fu, J. et al. Food intake regulates oleoylethanolamide formation and degradation in the proximal small intestine. J. Biol. Chem. 282, 1518-1528 (2007).

24. Desfossés, J., Stip, E., Bentaleb, L. A. \& Potvin, S. Endocannabinoids and schizophrenia. Pharmaceuticals 3, 3101-3126 (2010).

25. Silvestri, C. \& Di Marzo, V. The endocannabinoid system in energy homeostasis and the etiopathology of metabolic disorders. Cell Metab. 17, 475-490 (2013).

26. Pertwee, R. G. The pharmacology of cannabinoid receptors and their ligands: an overview. Int. J. Obes. 30, S13-S18 (2006). Suppl 1.

27. Frank, E. et al. Platform for systems medicine research and diagnostic applications in psychotic disorders-The METSY project. Eur. Psychiatry 50, 40-46 (2018)

28. Borgan, F. et al. The effects of cannabinoid 1 receptor compounds on memory: a meta-analysis and systematic review across species. Psychopharmacology (Berl.) 236, 3257-3270 (2019).

29. Hungund, B. L. et al. Upregulation of CB1 receptors and agonist-stimulated [35S] GTPgammaS binding in the prefrontal cortex of depressed suicide victims. Mol. Psychiatry 9, 184-190 (2004).

30. Neumeister, A. et al. Elevated brain cannabinoid CB1 receptor availability in posttraumatic stress disorder: a positron emission tomography study. Mol. Psychiatry 18, 1034-1040 (2013).

31. Hietala, J. 42.4 The endocannabinoid system in first-episode psychosis. Schizophr. Bull. 44, S69-S69 (2018). (Suppl 1).

32. Ranganathan, $M$. et al. Reduced brain cannabinoid receptor availability in schizophrenia. Biol. Psychiatry 79, 997-1005 (2016).

33. Borgan, F. et al. In vivo availability of cannabinoid 1 receptor levels in patients with first-episode psychosis. JAMA Psychiatry 76, 1074-1084 (2019).

34. Minichino, A. et al. Measuring disturbance of the endocannabinoid system in psychosis: a systematic review and meta-analysis. JAMA Psychiatry 76, 914-923 (2019).

35. Kaddurah-Daouk, R. et al. Metabolomic mapping of atypical antipsychotic effects in schizophrenia. Mol. Psychiatry 12, 934-945 (2007).

36. Oresic, M. et al. Metabolome in schizophrenia and other psychotic disorders: a general population-based study. Genome Med. 3, 19 (2011).

37. Holmes, E. et al. Metabolic profiling of CSF: evidence that early intervention may impact on disease progression and outcome in schizophrenia. PLoS Med. 3, e327 (2006)

38. Quinones, M. P. \& Kaddurah-Daouk, R. Metabolomics tools for identifying biomarkers for neuropsychiatric diseases. Neurobiol. Dis. 35, 165-176 (2009).

39. Oresic, M. et al. Metabolome in progression to Alzheimer's disease. Transl. Psychiatry 1, e57 (2011).

40. Bogdanov, M. et al. Metabolomic profiling to develop blood biomarkers for Parkinson's disease. Brain 131, 389-396 (2008). (Pt 2).

41. Foley, D. L. \& Morley, K. I. Systematic review of early cardiometabolic outcomes of the first treated episode of psychosis. Arch. Gen. Psychiatry 68, 609-616 (2011).

42. Henderson, D. C., Vincenzi, B., Andrea, N. V., Ulloa, M. \& Copeland, P. M. Pathophysiological mechanisms of increased cardiometabolic risk in people with schizophrenia and other severe mental illnesses. Lancet Psychiatry 2, 452-464 (2015).
43. Di Marzo, V. The endocannabinoid system in obesity and type 2 diabetes. Diabetologia 51, 1356-1367 (2008).

44. Jourdan, T., Godlewski, G. \& Kunos, G. Endocannabinoid regulation of beta-cell functions: implications for glycaemic control and diabetes. Diabetes Obes. Metab. 18, 549-557 (2016).

45. Leweke, F. M. et al. Cannabidiol enhances anandamide signaling and alleviates psychotic symptoms of schizophrenia. Transl. Psychiatry 2, e94-e94 (2012).

46. Fanelli, F. et al. Estimation of reference intervals of five endocannabinoids and endocannabinoid related compounds in human plasma by two dimensional-LC/ MS/MS. J. Lipid Res. 53, 481-493 (2012).

47. Mechoulam, R., Fride, E. \& Di Marzo, V. Endocannabinoids. Eur. J. Pharm. 359, 1-18 (1998).

48. Cravatt, B. F. et al. Molecular characterization of an enzyme that degrades neuromodulatory fatty-acid amides. Nature 384, 83 (1996)

49. Dinh, T. P. et al. Brain monoglyceride lipase participating in endocannabinoid inactivation. PNAS 99, 10819 (2002).

50. Rask-Andersen, M., Olszewski, P. K., Levine, A. S. \& Schiöth, H. B. Molecular mechanisms underlying anorexia nervosa: focus on human gene association studies and systems controlling food intake. Brain Res. Rev. 62, 147-164 (2010).

51. Engeli, S. et al. Activation of the peripheral endocannabinoid system in human obesity. Diabetes 54, 2838-2843 (2005).

52. Utevsky, A. V., Smith, D. V. \& Huettel, S. A. Precuneus is a functional core of the default-mode network. J. Neurosci. 34, 932-940 (2014).

53. Leech, R., Braga, R. \& Sharp, D. J. Echoes of the brain within the posterior cingulate cortex. J. Neurosci. 32, 215-222 (2012).

54. Rikandi, E. et al. Connectivity of the precuneus-posterior cingulate cortex with the anterior cingulate cortex-medial prefrontal cortex differs consistently between control subjects and first-episode psychosis patients during a movie stimulus. Schizophr. Res. 199, 235-242 (2018).

55. DiPatrizio, N. V. \& Piomelli, D. The thrifty lipids: endocannabinoids and the neural control of energy conservation. Trends Neurosci. 35, 403-411 (2012).

56. Boorman, E., Zajkowska, Z., Ahmed, R., Pariante, C. M. \& Zunszain, P. A. Crosstalk between endocannabinoid and immune systems: a potential dysregulation in depression? Psychopharmacology (Berl.) 233, 1591-1604 (2016).

57. Narayan, S., Head, S. R., Gilmartin, T. J., Dean, B. \& Thomas, E. A. Evidence for disruption of sphingolipid metabolism in schizophrenia. J. Neurosci. Res. 87, 278-288 (2009).

58. Bioque, M. et al. Peripheral endocannabinoid system dysregulation in firstepisode psychosis. Neuropsychopharmacology 38, 2568-2577 (2013).

59. Centonze, D., Battistini, L. \& Maccarrone, M. The endocannabinoid system in peripheral lymphocytes as a mirror of neuroinflammatory diseases. Curr. Pharm. Des. 14, 2370-2342. (2008)

60. Westerbacka, J. et al. Splanchnic balance of free fatty acids, endocannabinoids, and lipids in subjects with nonalcoholic fatty liver disease. Gastroenterology 139 1961-1971 e1961 (2010).

61. Oresic, M. et al. Prediction of non-alcoholic fatty-liver disease and liver fat content by serum molecular lipids. Diabetologia 56, 2266-2274 (2013).

62. Osei-Hyiaman, D. et al. Endocannabinoid activation at hepatic CB1 receptors stimulates fatty acid synthesis and contributes to diet-induced obesity. J. Clin Invest. 115, 1298-1305 (2005).

63. Woodhams, S. G., Sagar, D. R., Burston, J. J. \& Chapman, V. The role of the endocannabinoid system in pain. Handb. Exp. Pharm. 227, 119-143 (2015).

64. Guzmán, M. et al. Oleoylethanolamide stimulates lipolysis by activating the nuclear receptor peroxisome proliferator-activated receptor a (PPAR-a). J. Biol. Chem. 279, 27849-27854 (2004).

65. Fu, J. et al. Oleylethanolamide regulates feeding and body weight through activation of the nuclear receptor PPAR-alpha. Nature 425, 90-93 (2003).

66. Provensi, G. et al. Satiety factor oleoylethanolamide recruits the brain histaminergic system to inhibit food intake. Proc. Natl Acad. Sci. USA 111, 11527-11532 (2014).

67. Tam, J. et al. The therapeutic potential of targeting the peripheral endocannabinoid/CB1 receptor system. Eur. J. Intern. Med. 49, 23-29 (2018).

\section{ACKNOWLEDGEMENTS}

This project has received funding from the European Union's Seventh Framework Programme for project METSY-Neuroimaging platform for characterization of metabolic comorbidities in psychotic disorders (grant no. 602478). We thank to Aidan McGlinchey for assistance with editing the manuscript. We would like to thank the Turku Metabolomics Centre and Biocenter Finland for their support in the metabolomics analysis. We also wish to acknowledge the following METSY project investigators: Raimo K. R. Salokangas, Tuula Ilonen, Päivi Jalo, Akseli Mäkelä, Tiina From, Janina Paju, Anna Toivonen, Reetta-Liina Armio, Mirka Kolkka, Maija Walta, Max Karukivi, Juha Mäkelä, Maria Tikka, Olof Solin, Merja Haaparanta-Solin, Aidan McGlinchey, Juha Pajula, Mark van Gils, Juha M. Kortelainen, Carmen Moreno, Joost Janssen, Javier Santonja, Covadonga M. Diaz-Caneja, Miriam Ayora Rodriguez, Celso Arango, Alberto 
Rodriguez-Quiroga, Fabian Hernández-Álvarez, Jose L. Ayuso-Mateos, Roberto Rodriguez-Jimenez, Angela Ibañez, Jaana Suvisaari, Maija Lindgren, Teemu Mäntylä, Tuula Kieseppä, Outi Mantere, Eva Rikandi, Tuukka T. Raij, Dieter Maier, Elisabeth Frank, and Markus Butz-Ostendorf. Open access funding provided by Örebro University.

\section{AUTHOR CONTRIBUTIONS}

M.O., J.H., and O.H. initiated, designed, and supervised the study. A.M.D., T.R., T.L., and T.H. acquired serum endocannabinoid data by mass spectrometry. F.B., H.L., T.M., and M.V. acquired neuroimage data. A.M.D., F.B., H.L., and M.O. analyzed the data. A.M.D., S.L., and M.O. wrote the first draft of the manuscript. All authors approved the final version.

\section{COMPETING INTERESTS}

The authors declare no competing interests.

\section{ADDITIONAL INFORMATION}

Supplementary information is available for this paper at https://doi.org/10.1038/ s41537-020-00110-7.

Correspondence and requests for materials should be addressed to A.M.D. or M.O.
Reprints and permission information is available at http://www.nature.com/ reprints

Publisher's note Springer Nature remains neutral with regard to jurisdictional claims in published maps and institutional affiliations.

Open Access This article is licensed under a Creative Commons Attribution 4.0 International License, which permits use, sharing, adaptation, distribution and reproduction in any medium or format, as long as you give appropriate credit to the original author(s) and the source, provide a link to the Creative Commons license, and indicate if changes were made. The images or other third party material in this article are included in the article's Creative Commons license, unless indicated otherwise in a credit line to the material. If material is not included in the article's Creative Commons license and your intended use is not permitted by statutory regulation or exceeds the permitted use, you will need to obtain permission directly from the copyright holder. To view a copy of this license, visit http://creativecommons. org/licenses/by/4.0/.

(c) The Author(s) 2020 